\title{
Contributions of patient and citizen researchers to 'Am I the right way up?' study of balance in posterior cortical atrophy and typical Alzheimer's disease
}

Sebastian J. Crutch ${ }^{1,2}$, Keir X. X. Yong ${ }^{1,2}$, Amy Peters ${ }^{3}$, Dilek Ocal ${ }^{1}$, Diego Kaski ${ }^{3}$, Aida Suarez Gonzalez ${ }^{1}$, Natalie Ryan ${ }^{1}$, Simon Ball ${ }^{4}$, Charlie R. Harrison ${ }^{2,5}$, Charlie Murphy ${ }^{2,5}$, Pam Hulme ${ }^{6}$, Isabel Phillips ${ }^{6}$, Gaynor Hulme ${ }^{6}$, Andrew Brown ${ }^{6}$, Lu Brown ${ }^{6}$, Peter Riley ${ }^{7}$, Lynn Ramsey ${ }^{7}$, Anthony Woods ${ }^{8}$, Brian Day $^{2,3}$

${ }^{1}$ Dementia Research Centre, Institute of Neurology, University College London, London, UK

${ }^{2}$ Created Out of Mind, The Hub, Wellcome Collection, London, UK

${ }^{3}$ Sobell Department of Motor Neuroscience and Movement Disorders, UCL Institute of Neurology,

University College London, London, UK

${ }^{4}$ Filmmaker and visual artist, Berlin, Germany

${ }^{5}$ Visual Artist, London, UK

${ }^{6}$ Rare Dementia Support, London, UK

${ }^{7}$ Alzheimer's Society Lay Review Panel

${ }^{8}$ Wellcome, Euston Road, London, UK

Address for correspondence:

Sebastian Crutch

Dementia Research Centre, UCL Institute of Neurology

Box 16, National Hospital

Queen Square

London WC1N 3BG

s.crutch@ucl.ac.uk

\section{Abstract}

The current report describes the journey from the sharing of a single, extraordinary experience during a support group conversation to the development of a novel scientific investigation of balance problems in a rarer form of dementia. The story centres around the involvement of people living with or caring for someone with posterior cortical atrophy (PCA; often referred to as the 'visual variant of Alzheimer's disease) in highlighting hitherto under-appreciated consequences of their condition upon their ability to know 'Am I the right way up?'. We describe how comments and descriptions of these balance symptoms were collated and communicated, and the involvement of people with PCA in shaping a series of scientific hypotheses and developing and adapting appropriate experimental materials and procedures. We also reflect more broadly on how we might better recognise, acknowledge and encourage different forms of involvement, and describe several engagement-inspired extensions to the research involving people living with dementia, scientists and artists.

\section{Aims and background}

It is difficult to report the 'aim of PPI' per se in the current study as technically PPI preceded the study. The origins of the research - by which we mean the ideas and creative inspiration which led eventually to formal scientific hypotheses - lie in a broader ongoing relationship between people with posterior cortical atrophy (PCA), care partners, clinicians and researchers formed through our PCA Support Group (www.raredementiasupport.org/pca/). It was at one of these meetings in the Dementia Research Centre in September 2013 that Pam Hulme and Isabel Phillips related how their mother(-in-law) Gaynor Hulme had recently asked them, "Am I the right way up?". This comment was remarkable as posterior cortical atrophy (PCA), the condition with which Gaynor lived, had hitherto been known primarily for its characteristic progressive loss of visual processing (difficulty 
seeing what and where things are owing to degeneration of the visual cortex; an issue of 'brainsight' not eyesight; Benson et al., 1988; Crutch et al., 2017). Though not the first hint of non-visual sensory challenges in PCA (see Group discussions and Q\&As below), Gaynor's subjective experience strongly suggested a broader disruption of the way in which the balance system integrates different types of sensations. ${ }^{1}$

The only relevant previous investigation the DRC clinical research team had been involved in was triggered by another person with PCA describing how she had once experienced a complete $180^{\circ}$ room tilt illusion: "When I got downstairs the whole of the room was upside down, which was actually very scary but I got over that when I realized it was OK if you know what I mean. But it was completely reversed, the bottom was on the top and the top was on the bottom. Do you see what I mean? It was most peculiar." (Crutch et al., 2011, p12). However, to the best of our knowledge this experience only occurred once and has not been described by other people with PCA.

Therefore the primary aim of subsequent PPI activities was to understand whether Gaynor's experience was shared by others, and to gather descriptions of balance problems in PCA to help frame a scientific investigation of the phenomena.

\section{Methods and Results}

\section{Collating comments and descriptions of symptoms from group discussions and Q\&As}

Methods: The first action was to collate evidence of balance difficulties from previous group discussions, support group Q\&A sessions and clinical conversations. We retrospectively reviewed recordings of previous PCA Support Group meetings where relevant topics had been discussed. These included a talk and subsequent Q\&A by neuro-ophthalmologist Diego Kaski and neurologist Natalie Ryan on dizziness (March 2011; see references) and a further presentation by Natalie Ryan about unusual symptoms (October 2012; see references).

Results: The relaying of Gaynor's experience at the September 2013 meeting prompted another support group member in attendance to comment: "I just wanted to say something about posture because I also think that's a bit of a problem. [My former partner who has PCA] doesn't just shuffle but he's leaning to one side... and it's just very, very difficult, and you can't get him to stand up straight, and it also means helping him to get a jacket or coat on is that much more difficult."

The review of the 2011 and 2012 meetings revealed that complaints of "dizziness" were common, with a number of care partners also reporting people with PCA leaning to one side when walking or sitting with their head in their laps. It was also evident from one gentleman's comments that posture might be perceived to be imbalanced even when not obviously misaligned in the physical world; he described that when walking along a pavement, "I felt like I was about to fall off the edge of the world".

These reports were consistent with our informal review of clinical experience amongst colleagues prompted by Gaynor's remark, who described consequences of dysfunctional balance in support group members and research participants which had been intimated through reports of being less likely to leave the home due to fear of falling. For example, some participants described being particularly hesitant to leave the home after rainfall (not only because surfaces were more slippery, but also because of interference with perceiving the depth of reflective surfaces), considerably reduced mobility, and injuries arising from falls.

\section{Connecting people with relevant expertise}

\footnotetext{
${ }^{1}$ The balance system relies upon sensory information to relate body position to the gravitational vertical and to report how the body is moving to stop you falling over. Visual, vestibular (from inner ear) and proprioceptive (from muscles and joints) information all play a role but no one sensory system on its own provides the brain with the complete story. Instead, the information from all three has to be combined. Combining these types of information is known to require the parietal lobes of the brain which are particularly vulnerable in PCA and typical AD.
} 
Methods: The next step in the development of the research project was to bring members of the PCA support group with a lived experience of balance problems into conversation with experts in the neuroscience of balance.

Results: Support group members gave verbal consent for their quotes to be shared with Brian Day and Amy Peters from UCL's Sensorimotor Laboratory in Queen Square. These comments and descriptions were invaluable for familiarising these researchers, who had not worked previously with individuals with PCA, with the condition.

\section{Pilot testing and feedback}

Methods: Pilot testing was arranged, with PCA support group members Andrew and Lu Brown attending the Sensorimotor Laboratory on two occasions (March and April 2014) to participate in preliminary discussions and investigations. Subsequent minor modifications to stimuli and apparatus were made following input from other participants early in the testing phase.

Results: Andrew completed pilot testing of several tasks including the manipulation of sense of balance by rotating visual dots (see Figure 1). Andrew's performance on existing experimental paradigms informed the scientific hypotheses. For example, it became apparent that it would be necessary not just to test standing balance but also visual perception of the vertical when seated.

His interactions with the test equipment also determined which response measures (e.g. verbal report, button rotation, manipulation of a response bar) might be most appropriate for individuals with PCA. Observation and explicit feedback triggered a number of specific modifications to the testing apparatus e.g.

- Use of a larger knob for controlling the rod in rod-and-frame test (during which participants try to maintain the verticality of a rod within a vertical or learning frame). The standard smaller knob was too difficult to manipulate, partly owing to the co-occurrence of apraxia in PCA.

- Introduction of a soft head brace to create a fixed head position during the rod and frame, rod and dot motion tests.

- The original rod in the rod and frame test consisted of individual blobs but this was not appropriate because of PCA patients' problems with perceiving fragmented figures as whole forms. In the same regard, the frame was originally just an outline but elements of this frame were misperceived as being the target rod. Consequently the frame was exchanged for a filled grey shape (circle or rectangle) to make it more continuous but maintain the contrast of light rod against a dark background. This required complete re-programming of the rod and frame experiment to generate a non-pixelating stimulus rod and the new frame, plus adjusting the size of the stimulus to account for participants' reduced effective field of vision (Crutch et al., 2011).

- We created an apparatus to provide tactile and visual assistance when positioning feet for standing tasks in response to participants having considerable difficulty standing on a marked position on the floor for trials.

Andrew's descriptions of experiences also led to the introduction of additional paradigms into the planned project. For example, his memorable statement "I sat on my vicar" (in reference to a dinner party when, on returning to the table from the bathroom, he had misjudged the location of his empty chair and had accidentally sat in the lap of the neighbouring guest) led us to measure directly people's movements and accuracy in manoeuvring their body to sit on a target location on a bench. 

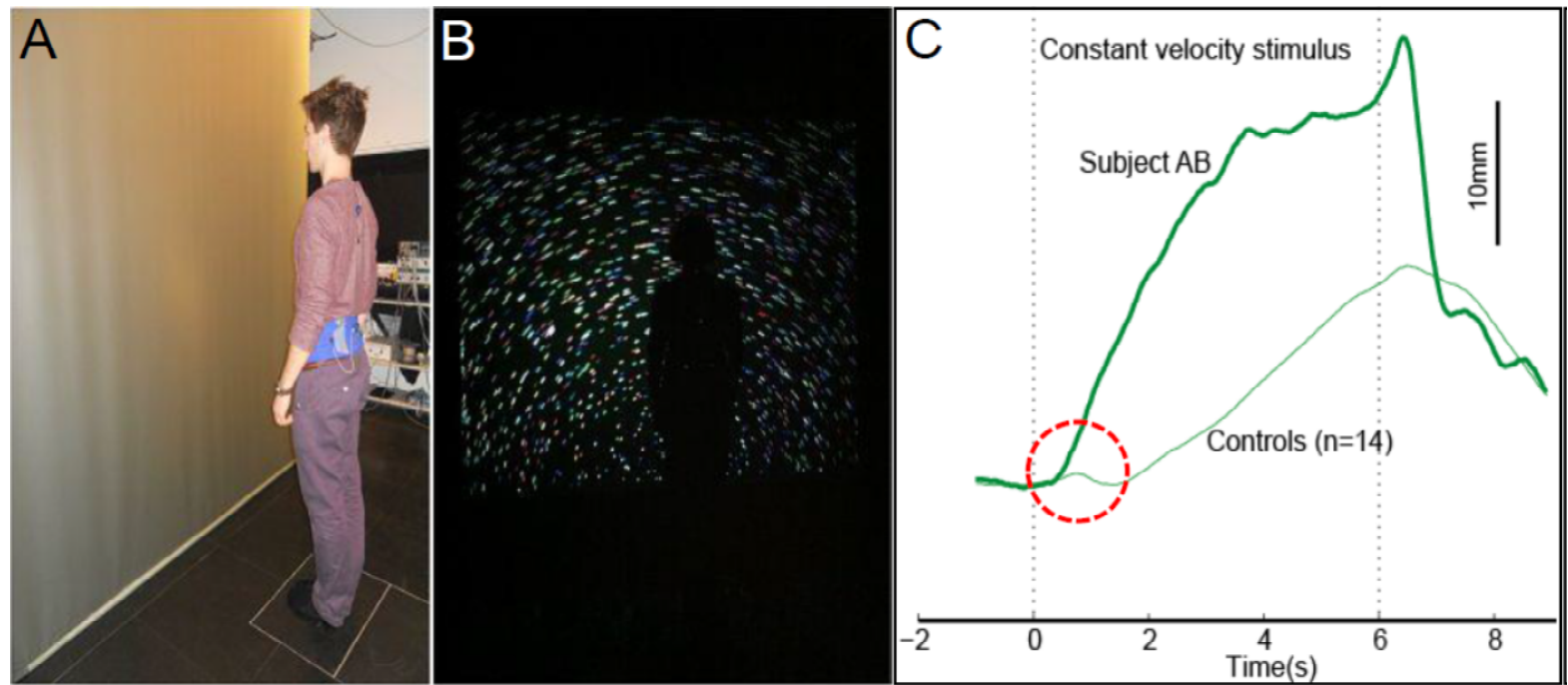

Figure 1. Visual perturbations - impact of visual motion on perception and standing balance. This experiment evaluates whether visual motion (rotating dots) can be misinterpreted by people with dementia as indicating self-movement ("I'm moving") rather than movement of the world ("the dots are moving"). The participant stands on force plates in front of a projection screen (see Figure 1a). A rotating visual scene is presented, consisting of dots moving at constant acceleration or velocity (see 1b). The direction, speed and acceleration of the dots are varied and the resulting lean to either side and change in pressure on the feet measured. In our preparatory studies (see 1c), Andrew (thick line) showed abnormally large leaning responses relative to controls (thin line) when presented with moving dots. Unlike the controls, Andrew also showed no initial correction of this lean (dotted red circle). This pilot experiment indicated possible damage to a specific mechanism that makes rapid decisions about whether the self or the world is moving, which is key to preventing leaning and falls.

\section{Review of funding application, ethics application and annual Research Network visit}

Methods: People with PCA and care partners reviewed the funding application and ethics application documents. The Alzheimer's Society grant review process also involved feedback from the Research Network comprising people living with or caring for someone with dementia. Once funded, the research team also participated in annual monitoring visits from representatives of the Research Network.

Results: The project received funding from the Alzheimer's Society, in part owing to the level of patient and carer involvement in the development of the project. Despite PCA being a relatively rare condition, we were fortunate that one visiting member of the Research Network who himself lives with PCA subsequently participated in the experiment. No payments were made to non-UCL staff team members, but travel and subsistence expenses related to study participation and PPI activities were paid.

\section{Review of participant information sheets and consent forms}

Methods: A substantial amendment to existing study ethics was drafted in 2016, prompted largely by feedback from participants with PCA on the consenting process. A proportion of patients demonstrated considerable difficulty with reading information sheets and/or writing on consent forms due to visual processing and coordination difficulties. Consequently, core features of the amended study documents and protocol included approaches to maximise accessibility of study documents and consenting procedures for participants with PCA, as well as separating the overall study into sub-studies to facilitate interpretability of what participants would be asked to do on different research visits. A care partner reviewed draft patient information sheets, consent forms and study protocol (May 2016). 
Results: The amendment formalised how to present information sheets, support participants negotiating consent forms with multiple boxes requiring written responses, and provided the option of verbal audio-recorded consent. Benefits of the care partner review included reducing unnecessary application of terminology within information sheets/consent forms (e.g. use of 'pair' rather than 'diad').

6. Involvement in engagement, and engagement-inspired extensions to the research

Methods: Opportunities for engaging a variety of audiences with the topic were apparent early in project development, with the funding application including support for film maker Simon Ball to create an animation based on interviews with people with PCA and their partners. The advent of the Created Out of Mind dementia and arts residency at The Hub, Wellcome Collection, also yielded opportunities for people with PCA to share their subjective experiences of the condition and of the balance testing sessions with artists including Charles Harrison and Charlie Murphy.

Results: Inspired by six personal stories, the resulting animated film 'Do I See What You See?' recreates what it feels like to live with the knowledge that your brain might be playing tricks on you and you are seeing differently from everyone else. The film can be viewed at:

http://www.createdoutofmind.org/stories-and-reflections/do-i-see-what-you-see . The study also inspired a number of public events, workshops, student collaborations and displays (see Figures and 3 ) and a novel arts-science experiment in the form of a painted gestural equivalent of the balance study's rod-and-frame verticality test (Figure 2).
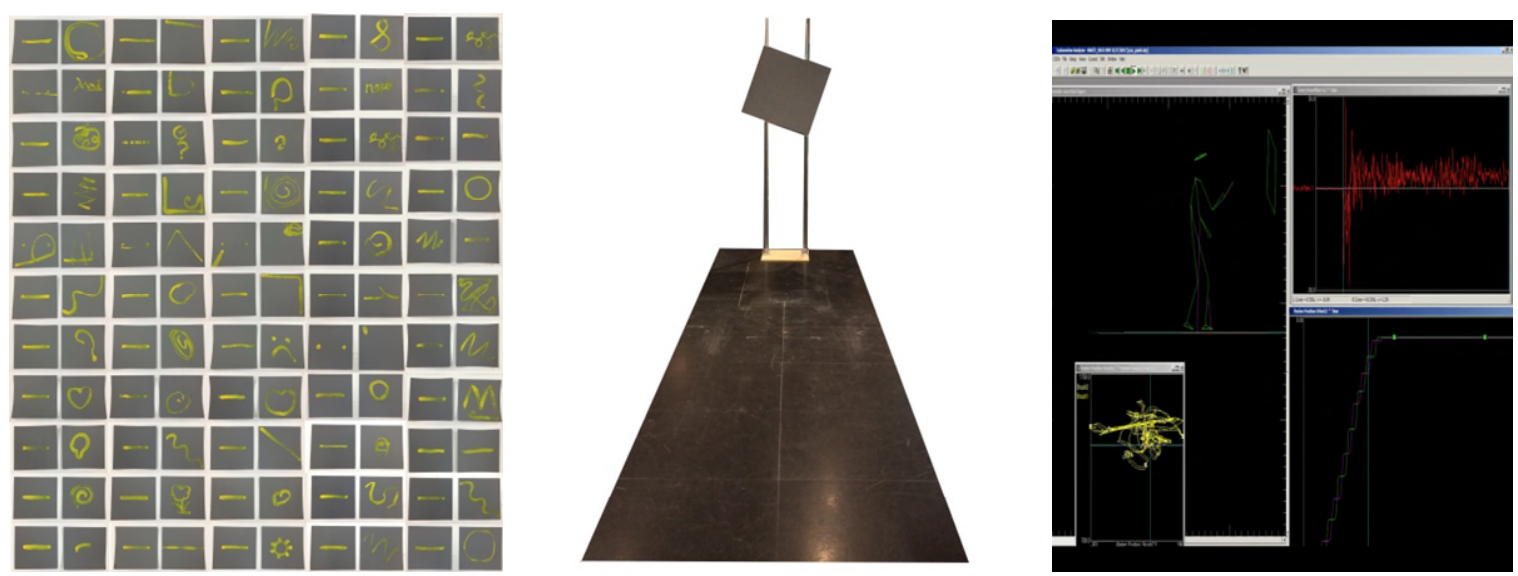

Figure 2. Left panel: Single Yellow Lines, Charles Harrison's painting-based exploration of 'in the moment' experience in which PCA and other support group members were invited to paint two single lines, one straight and one of their choosing. Middle and right panels: Collaborative research between Harrison and Prof Brian Day into controlled and creative painted gestures made at UCL Sensorimotor Lab. 


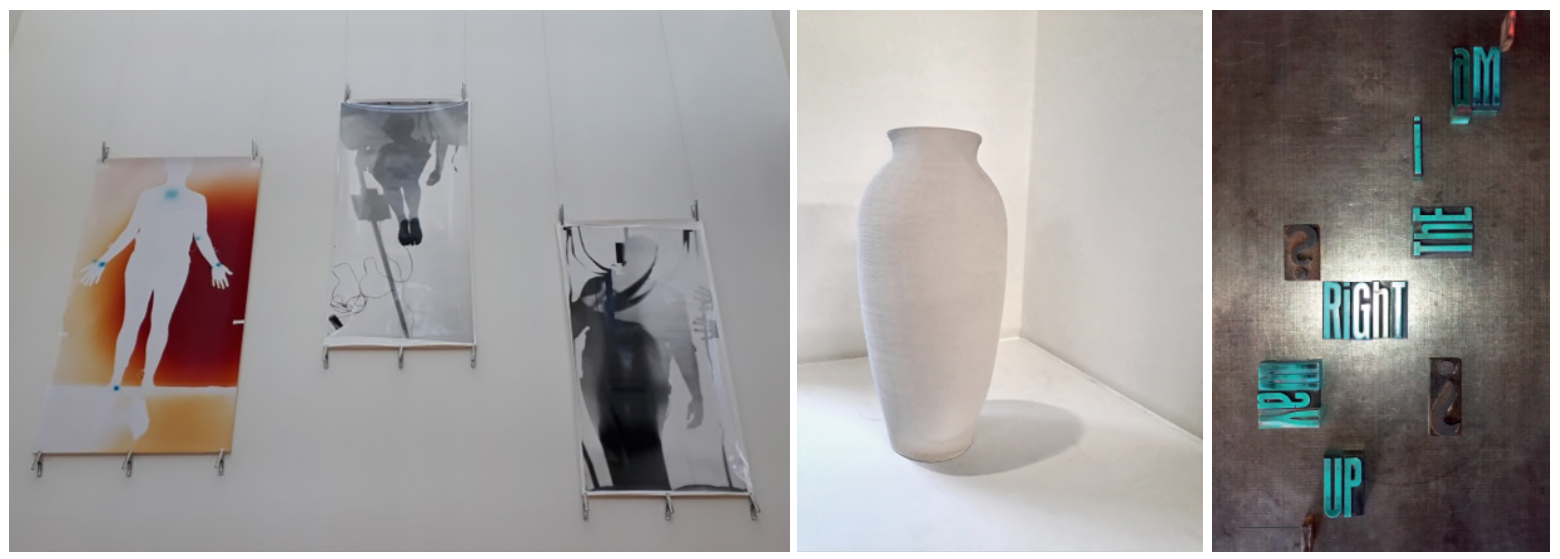

Figure 3. Left panel: Charlie Murphy's 'balance' photograms investigating participants' experiences of self-doubt, disorientation and suspension during balance tests, in the Trajectories exhibition at LifeSpace Science Art Research Gallery, Dundee (2 February - 2 June 2018;

http://lifespace.dundee.ac.uk/exhibition/trajectories). Middle panel: Illusion Vase by Rachel Wilcock (from Central St Martins' BA Ceramic Design collective Studio Senses) using 3d modelling software to visualise changes in perception of shape and orientation from different angles. Right panel: Murphy's typographic experiments with Gaynor's statement provided the stimulus for participatory letter press workshops with people with dementia and care partners during 2017 Dementia Awareness Week.

\section{Discussion}

In the current report, we highlight the crucial role of people living with rarer dementia syndromes such as PCA in raising awareness not only of their condition as a whole but specifically of often under-recognised characteristics and consequences of that condition. The involvement of people with PCA was important at every stage of the investigation, not least in triggering the whole study (see further discussion of 'inspiration' below). As the topic of balance in PCA had not been examined previously, without the involvement of people from the earliest stages, it would not have been possible to develop a scientifically appropriate, rigorous or valid approach to these problems. This critical input was particularly highlighted by the numerous and extensive changes made to the test materials and procedures during and following pilot testing without which the data collected from subsequent participants would have been noisy, unreliable and in some cases simply unobtainable.

PCA involvement in this project was greatly facilitated through links with the PCA support group and the fact that some participants are known through repeated visits to contribute to our PCA research programme over multiple years. Such participants and carers have ended up carrying out roles through which we as researchers work with them in a capacity that differs from a typical researcher-patient-carer dynamic (e.g. involvement as participant + citizen researchers, regional support group facilitators, advisory committee members).

Given the central importance of the lived experience of balance problems to the current study, arguably one limitation is that we did not systematically collect audio recorded debriefing data from participants. During other investigations in simulated environments (e.g. Yong et al., in press), participants have worn mobile eyetrackers which collect the comments, reactions and reflections of participants during or between trials. However there are clear trade-offs here between wishing to maximize the opportunity to learn from participant experiences and not wishing to overcomplicate already complex paradigms or to overburden participants with extra tasks during research visits.

It may also have been ideal to record participants articulating why disordered balance, falls and fear of falls are meaningful at an individual level, in a similar way to when we carried out studies with people with acquired dyslexia due to PCA who mentioned it meant they could not read to grandchildren or placed it within the same bracket as losing the ability to drive. As an example regarding balance and gait disturbances, the wife of an familial AD participant in another study described her husband as having to move himself down stairs at home while sitting as he did not feel 
comfortable walking down. We did not systematically ask participants in the current study for examples of the everyday impact of their balance problems, although many of the current participants had already contributed to a separate qualitative, semi-structured interview-based study of living with PCA (Harding et al., 2018).

The richness of the experiences shared by people living PCA before and during the study are reflected not just in the variety of experimental tasks already administered or which evolved during the study (see 'Extensions to the research' below), but also in plans for future investigations. A number of additional behavioural phenomena have been described or observed which will guide future experimental investigation into sitting and standing dynamics and into behavioural strategies to support people experiencing dementia-related balance problems. Owing to time constraints during research visits, it has also not yet been possible to follow up on all tasks considered during pilot testing, such as the influence of balance problems upon walking behaviour when approaching obstacles or narrow openings. In this way, the contributions already made by people with or caring for someone with PCA during this study are not limited in their usefulness or importance to the timeframe of the current project.

\section{Reflections and critical perspectives}

Recommendations for recognising, acknowledging and encouraging different forms of involvement The central importance of Gaynor, Pam and Isabel's comments in shaping the current research project is for us an example of involvement by inspiration. Put simply, the whole research project can be traced back to a single statement, which was then affirmed and elaborated upon by other members of the PCA support group. This and other similar experiences of the many and varying contributions to research of patient and citizen researchers have led us to recently propose recommendations for how these contributions should be recognised, acknowledged and encouraged (Crutch et al., submitted). These contributions include:

1. Inspiration: generating new ideas and hypotheses, and challenging existing assumptions.

2. Context setting and training: opening up the lived experience to other researchers.

3. Project design and development: co-design, revision, feasibility assessment and piloting as an active patient/citizen researcher.

4. Motivation and insights through participation in established projects.

5. Review, reflection and rethinking: scrutinising current work and improving future research.

Communicating the crucial role of people with dementia in shaping research about the conditions they live with may facilitate the involvement of people affected by other health conditions in which $\mathrm{PPI}$ is less established.

\section{Summary}

The contributions of people living with dementia and their supporters have given rise to a project that seeks to improve understanding, detection and management of balance problems in PCA, and provide a rounded account of its impact on people's lives. Members of the PCA Support Group and others should be acknowledged not just for participating in the research, but for inspiring the project, setting the context, providing feedback which shaped the design of experiments, and reviewing and reflecting upon the research as a whole.

\section{Lessons for practice: What worked and what would we recommend to others}

1. People living with dementia or supporting someone who is can influence research in a variety of ways including inspiring the project, setting the context, providing feedback which shapes the design of experiments and reviewing and reflecting upon the research as a whole.

2. These varying and creative contributions should be clearly acknowledged and encouraged.

3. PPI can occur not just in committees and working groups formed specifically for the purpose, but also prospectively through broader ongoing conversations and relationships between people 
with posterior cortical atrophy (PCA), care partners, clinicians and researchers in a variety of settings.

4. PPI provides a critical mechanism for enhancing research through consideration of the subjective experience of a health condition.

\section{Acknowledgements}

This article is dedicated in loving memory to Gaynor Hulme (1948-2018). We are grateful to many members of the PCA Support Group for conversations about issues of balance in everyday life. This work was supported by an Alzheimer's Society project grant (AS-PG-14-022). SC, SB, CH and CM also receive support from Wellcome (200783). The Dementia Research Centre receives support the NIHR Queen Square Dementia BRU and the NIHR UCL/H Biomedical Research Centre.

\section{References}

Benson DF, Davis RJ, Snyder BD. Posterior cortical atrophy. Archives of Neurology. 1988 Jul;45(7):789-93.

Crutch SJ, Lehmann M, Gorgoraptis N, Kaski D, Ryan N, Husain M, Warrington EK. Abnormal visual phenomena in posterior cortical atrophy. Neurocase 2011, 17 (2), 160-177.

Crutch SJ, Herron D...Rossor MN. Recommendations for acknowledging the contributions to research of people with dementia and their carers. Submitted.

Crutch SJ, Schott JM, Rabinovici GD, Murray M, Snowden JS, van der Flier WM, Dickerson BC, Vandenberghe R, Ahmed S, Bak TH, Boeve BF, Butler C, Cappa SF, Ceccaldi M, de Souza LC, Dubois B, Felician O, Galasko D, Graff-Radford J, Graff-Radford NR, Hof PR, Krolak-Salmon P, Lehmann M, Magnin E, Mendez MF, Nestor PJ, Onyike CU, Pelak VS, Pijnenburg Y, Primativo S, Rossor MN, Ryan NS, Scheltens P, Shakespeare TJ, Suárez González A, Tang-Wai DF, Yong KXX, Carrillo M, Fox NC; Alzheimer's Association ISTAART Atypical Alzheimer's Disease and Associated Syndromes Professional Interest Area. Consensus classification of posterior cortical atrophy. Alzheimer's \& Dementia. 2017 Aug;13(8):870-884.

Harding E, Sullivan MP, Woodbridge R, Yong KXX, McIntyre A, Gilhooly ML, Gilhooly KJ, Crutch SJ. 'Because my brain isn't as active as it should be, my eyes don't always see': a qualitative exploration of the stress process for those living with posterior cortical atrophy. BMJ Open. 2018, 8(2):e018663. doi: 10.1136/bmjopen-2017-018663.

PCA Support Group newsletter (June 2011). www.ucl.ac.uk/drc/pcasupport/newsletter/newsletterpdfs/pca newsletter jun2011.pdf (accessed 01 February 2018).

Presentation by Natalie Ryan to PCA Support Group (26 October 2012). www.youtube.com/watch?v=tvP EsS5jyl (accessed 01 February 2018). 